\title{
Atlas trigger for first physics and beyond
}

\section{Teresa FONSECA MARTIN*}

Royal Holloway University of London, London

E-mail: teresa.fonseca.martindcern.ch

\section{On behalf of the ATLAS TDAQ Community}

The full author list is given in the Appendix and in ATL-DAQ-PUB-2008-004

\begin{abstract}
ATLAS is a multi-purpose spectrometer built to perform precision measurements of Standard Model parameters and is aiming at discovery of Higgs particle, Super Symmetry and possible other physics channels beyond Standard Model. Operating at $14 \mathrm{TeV}$ center of mass energy ATLAS will see 40 million events per second at nominal luminosity with about 25 overlapping interactions. Most of the events are inelastic proton-proton interactions with only few $\mathrm{W}, \mathrm{Z}$ bosons or ttbar pairs produced each second, and expectations for Higgs or SUSY production cross-section are much smaller than that. ATLAS trigger has a difficult task to select one out of $10^{5}$ events online and to ensure that most physics channels of interests are preserved for analysis. In this talk we will review the design of ATLAS trigger system, the trigger menu prepared for initial LHC run as well as for high luminosity run. The expected trigger performance of the base-line ATLAS physics programs will be reviewed and first results from the commissioning period will be given. The methods to measure trigger efficiencies and biases directly from data will be discussed.
\end{abstract}

Physics at LHC 2008

29 September - October 4, 2008

Split, Croatia

${ }^{*}$ Speaker. 


\section{Introduction, the ATLAS trigger}

ATLAS is a general purpose particle physics detector which is described in [1] and [2]. It will detect proton-proton collisions in the Large Hadron Collider (LHC). Its primary goals are to understand the mechanism for electroweak symmetry breaking and to search for new physics beyond the Standard Model. The LHC will eventually provide proton-proton collisions at a center of mass energy of about $14 \mathrm{TeV}$, a design luminosity of $10^{34} \mathrm{~cm}^{-2} \mathrm{~s}^{-1}$ and a bunch-crossing rate of $40 \mathrm{MHz}$. Due to the limits on our capability to store data the ATLAS trigger is expected to have a rejection power of $\sim 10^{5}$ while being efficient for the interesting events.

ATLAS trigger is structured in three levels. The First Level (L1) uses custom built electronics. It reduces the trigger rate in $\sim 3$ orders of magnitude, giving a response in less than $2.5 \mu \mathrm{s}$ It receives coarse data from the Calorimeter and muon sub-detectors. The so-called High Level Trigger (HLT) is software-based, it is composed by a Second Level Trigger (L2) and a third level trigger called Event Filter (EF). HLT has access to the full detector granularity data inside a Region of Interest (RoI) that has been defined by the previous trigger level. L2 uses algorithms specifically written for the trigger to accomplish the hard speed limits required at this level. EF uses offline reconstruction algorithms as much as possible in the online trigger environment.

\section{The ATLAS Trigger Menu \& Performance}

The ATLAS trigger menus are built based in the identification of potential candidates for interesting objects as: electrons, photons, taus, muons, jets, b-tagged jets, B-physics objects, missing transverse energy, etc.

Inclusive triggers for each of these objects are included in the menu as much as they are allowed by rate limitations. Main handle to limit rates are the selection of events above a given transverse energy $\left(E_{T}\right)$ threshold and the possibility to apply isolation criteria. The trigger menus also contain more sophisticated signatures in which two or more of the previous mentioned trigger objects are combined (for example: tau + missing $E_{T}$ signature). Detailed studies of trigger performance based on signal efficiency and background rejection have been performed using full detector Monte Carlo simulation. For details check the trigger chapter in Ref. [3], as an example, the trigger efficiency with respect to offline reconstructed objects versus their transverse energy is shown for muon triggers in Fig. 1]

\section{Trigger efficiency determination from data}

Methods are being developed to determine trigger efficiency from data, minimizing dependencies on Monte Carlo simulation. This is crucial for analysis, trigger data taking monitoring as well as Monte Carlo simulation validation and tuning. The key of these methods is to define a 'clean' data sample of a specific particle to be used as a reference to determine trigger efficiency for the corresponding trigger object. A typical example is the use of $Z \rightarrow e^{+} e^{-}$and $Z \rightarrow \mu^{+} \mu^{-}$decays. The control sample is defined by one lepton trigger plus the offline reconstruction of both leptons satisfying the $Z$ invariant mass requirement. The second lepton is then used to study the trigger efficiency performance [3]. Studies are ongoing to extrapolate this measured trigger efficiency to 


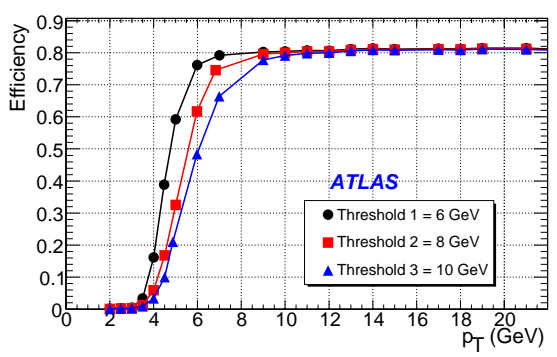

Figure 1: Trigger efficiency versus transverse momentum $\left(p_{T}\right)$ curves for three of the lower muon trigger $p_{T}$ thresholds.

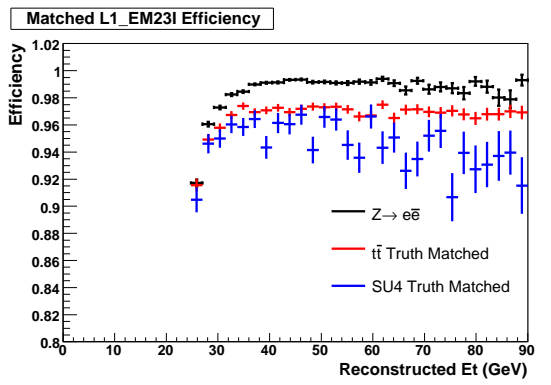

Figure 2: Comparison of L1 isolated electron trigger efficiency as a function of $E_{T}$ for different Monte Carlo simulation samples $\left(Z \rightarrow e^{+} e^{-}\right.$, ttbar and susy).

different event topologies, as can be seen in Fig. 2 Trigger efficiency will also be determined with respect to offline identified objects using as control samples 'Boot-strap' techniques (use looser trigger selections to study performance of tighter ones) and 'Orthogonal' triggers (Ex. use muon trigger to study performance of missing $E_{T}$ trigger).

\section{Commissioning}

The trigger system is now continuously used during cosmic ray runs to exercise the full trigger chain and read-out of sub-detectors. Since few months the L1 central trigger has been running in its final configuration receiving all foreseen trigger inputs and providing trigger signals to all subdetectors. HLT is periodically integrated in the cosmic runs. ATLAS received the first single beam in September 2009. The proton bunch sent onto the beam collimators originated the very first beam events to trigger on. Next phase was to have beam circulating for several turns. The time alignment for different trigger items was studied taking the Beam Pick-ups trigger as the time reference of the passage of a bunch in the ATLAS detector. The L1 decision of each trigger item in a time window of \pm 15 bunch crossing has been recorded and analyzed.

\section{Conclusions}

The ATLAS trigger is on a good track to have a successful performance for proton collisions at LHC. Methods are being developed to determine trigger efficiency from data. The full trigger system is used in cosmic ray runs. The L1 trigger performed successfully with single beam.

\section{References}

[1] Jenni, P et al 1999 ATLAS detector and physics performance Technical Design Report, ser. Technical Design Report ATLAS (Geneva: CERN)

[2] The ATLAS Collaboration, G. Aad et al., "The ATLAS Experiment at the CERN Large Hadron Collider”, JINST 3 (2008) S08003

[3] ATLAS Collaboration, "Expected Performance of the ATLAS Experiment, Detector, Trigger and Physics” CERN-OPEN-2008-020, Geneva, 2008 


\section{A. The ATLAS TDAQ Community}

Abolins M., ${ }^{56}$, Achenbach R. ${ }^{31}$, Adorisio C., ${ }^{15}$, Adragna P., ${ }^{76}$, Aharrouche M., ${ }^{57}$, Aielli G., ${ }^{81,42}$, Al-Shabibi A., ${ }^{31}$, Aleksandrov I., ${ }^{24}$, Alexandre G. ${ }^{26}$, Aloisio A., ${ }^{68,38}$, Alviggi M.G.. ${ }^{68,38}$, Amorim A., ${ }^{49}$, Amram N., ${ }^{88}$, Anderson K., ${ }^{16}$, Andrei V., ${ }^{31}$, Anduaga X., ${ }^{50}$, Antonelli S., ${ }^{10,36}$, Anulli F. ${ }^{80,41}$, Aracena I., ${ }^{82}$, Ask S., ${ }^{58}$, Åsman B., ${ }^{86}$, Avolio G., ${ }^{91}$, Backlund S., ${ }^{14}$, Badescu E., ${ }^{11}$, Baines J., ${ }^{77}$, Banerjee S., ${ }^{98}$, Barnett B.M., ${ }^{77}$, Bartoldus R., ${ }^{82}$, Batraneanu S., ${ }^{12,14}$, Battaglia A., ${ }^{7}$, Bauss B., ${ }^{57}$, Beauchemin P., ${ }^{72}$, Beck H.P., ${ }^{7}$, Bee C., ${ }^{59}$, Behera P., ${ }^{45}$, Bell P., ${ }^{58}$, Bell W.H., ${ }^{28}$, Bellagamba L., ${ }^{10,36}$, Bellomo M., ${ }^{39}$, Ben Ami S., ${ }^{87}$, Bendel M., ${ }^{57}$, Benhammou Y., ${ }^{88}$, Benslama K., ${ }^{78}$, Berge D., ${ }^{14}$, Berger N., ${ }^{48}$, Bernius C., ${ }^{92}$, Berry T., ${ }^{34}$, Bianco M.,${ }^{52,37}$, Biau C.,${ }^{95}$, Biglietti M. ${ }^{68,38}$, Bizjak I., ${ }^{92}$, Blair R.E., ${ }^{1}$, Bogaerts A., ${ }^{14}$, Bohm C., ${ }^{86}$, Boisvert V., ${ }^{34}$, Bold T., ${ }^{91,20}$, Bondioli M., ${ }^{91}$, Booth J.R.A., ${ }^{8}$, Borer C., ${ }^{7}$, Boscherini D., ${ }^{10,36}$, Bosman M., ${ }^{5}$, Boyd J., ${ }^{14}$, Bracinik J., ${ }^{8}$, Brawn I.P., ${ }^{77}$, Brelier B., ${ }^{62}$, Bressler S., ${ }^{87}$, Brock R., ${ }^{56}$, Brooks W.K., ${ }^{97}$, Brunet S., ${ }^{21}$, Bruni A., ${ }^{10,36}$, Bruni G., ${ }^{10,36}$, Bucci F., ${ }^{26}$, Buda S., ${ }^{11}$, Burckhart-Chromek D., ${ }^{14}$, Buttar C., ${ }^{28}$, Camarri P., ${ }^{81,42}$, Campanelli M., ${ }^{92}$, Canale V., ${ }^{68,38}$, Capasso L., ${ }^{68,38}$, Caprini M., ${ }^{11}$, Caracinha D., ${ }^{53}$, Caramarcu C., ${ }^{11}$, Cardarelli R., ${ }^{81,42}$, Carlino G., ${ }^{38}$, Casadei D., ${ }^{65}$, Casado P., ${ }^{5}$, Cataldi G., ${ }^{37}$, Cerri A., ${ }^{14}$, Charlton D.G., ${ }^{8}$, Childers J.T., ${ }^{31}$, Chiodini G. ${ }^{52,37}$, Ciapetti G., ${ }^{80,41}$, Cimino D., ${ }^{40}$, Ciobotaru M., ${ }^{91,12}$, Coccaro A., ${ }^{27}$, Collins N.J., ${ }^{8}$, Conde Muino P., ${ }^{49}$, Conventi F., ${ }^{38}$, Corradi M.., ${ }^{10,36}$, Corso-Radu A.,${ }^{91}$, Costa M.J.,${ }^{96}$, Coura Torres R.,${ }^{79}$, Cranmer K., ${ }^{65}$, Crone G.,${ }^{92}$, Crupi R.,${ }^{52,37}$, Cuenca Almenar C., ${ }^{91}$, Curtis C.J., ${ }^{8}$, Czyczula Z., ${ }^{64}$, Dam M., ${ }^{64}$, Damazio D.,${ }^{4}$, Darlea G.L., ${ }^{14}$, Davis A.O.,${ }^{77}$, De Pedis D., ${ }^{80,41}$, De Santo A., ${ }^{34}$, de Seixas J.M., ${ }^{79}$, DeAsmundis R., ${ }^{38}$, Degenhardt J., ${ }^{75}$, della Volpe D., ${ }^{68,38}$, DellaPietra M. ${ }^{68,38}$, Delsart P.-A., ${ }^{62}$, Demers S., ${ }^{82}$, Demirkoz B., ${ }^{14}$, Di Mattia A., ${ }^{56}$, Di Nardo R., ${ }^{81,42}$, Diaz M., ${ }^{84}$, DiCiaccio A., ${ }^{81,42}$, Dionisi C., ${ }^{80,41}$, Djilkibaev R., ${ }^{65}$, Dobson E., ${ }^{72}$, Dobson M., ${ }^{14}$, Dogaru M.,${ }^{11}$, dos Anjos A., ${ }^{100}$, Dotti A.., ${ }^{40}$, Dova M.T.,${ }^{50}$, Drake G., ${ }^{1}$, Dufour M.-A., ${ }^{60}$, Eckweiler S., ${ }^{57}$, Ehrenfeld W., ${ }^{30,21}$, Eifert T., ${ }^{26}$, Eisenhandler E. ${ }^{76}$, Ellis N., ${ }^{14}$, Emeliyanov D., ${ }^{77}$, Enoque Ferreira de Lima D., ${ }^{79}$, Ermoline Y., ${ }^{56}$, Etzion E., ${ }^{88}$, Falciano S., ${ }^{41}$, Farthouat P., ${ }^{14}$, Faulkner P.J.W., ${ }^{8}$, Fedorko A., ${ }^{14}$, Fedorko I., ${ }^{14}$, Feng E., ${ }^{16}$, Ferland J., ${ }^{62}$, Ferrag S., ${ }^{28}$, Ferrari R., ${ }^{74}$, Ferrer M.L., ${ }^{25}$, Fischer G., ${ }^{21}$, Flacher H., ${ }^{14}$, Fleckner J.E., ${ }^{57}$, Flowerdew M., ${ }^{54}$, Föhlisch F., ${ }^{31}$, Fonseca-Martin T., ${ }^{34}$, Francis D. ${ }^{14}$, Fratina S., ${ }^{75}$, Fukunaga C.,${ }^{90}$, Gadomski S., ${ }^{26}$, Gallacher M.P., ${ }^{8}$, Garitaonandia Elejabarrieta H., ${ }^{69}$, Gaudio G., ${ }^{74}$, Gee C.N.P., ${ }^{77}$, George S., ${ }^{34}$, Ghete V.M., ${ }^{44}$, Giagu S., ${ }^{80,41}$, Gillman A.R., ${ }^{77}$, Giusti P., ${ }^{10,36}$, Goebel M., ${ }^{21}$, Gonçalo R., ${ }^{34}$, Gonzalez Pinto F., ${ }^{13}$, Gonzalez Silva L., ${ }^{13}$, Göringer C., ${ }^{57}$, Gorini B., ${ }^{14}$, Gorini E., ${ }^{52,37}$, GrabowskaBold I., ${ }^{91,20}$, Grancagnolo S., ${ }^{35}$, Green B., ${ }^{34}$, Groll M., ${ }^{57}$, Guida A., ${ }^{52,37}$, Haas S., ${ }^{14}$, Hadavand H., ${ }^{83}$, Hadley D.R., ${ }^{8}$, Haller J., ${ }^{30,21}$, Hamilton A., ${ }^{26}$, Hanke P., ${ }^{31}$, Hansen J.R., ${ }^{64}$, Hasagawa S., ${ }^{67}$, Hasegawa Y., ${ }^{85}$, Hauschild M., ${ }^{14}$, Hauser R., ${ }^{56}$, Hayakawa T., ${ }^{47}$, Head S., ${ }^{58}$, Heijboer A., ${ }^{14}$, Heim S., ${ }^{56}$, Hellman S., ${ }^{86}$, Hershenhorn A., ${ }^{87}$, Hidvégi A., ${ }^{86}$, Hillier S.J., ${ }^{8}$, Hirayama S., ${ }^{89}$, Hod N., ${ }^{88}$, Hori T., ${ }^{47}$, Hryn'ova T., ${ }^{48}$, Hughes-Jonesa R. ${ }^{58}$, Huston J., ${ }^{56}$, Iacobucci G.. ${ }^{68,38}$, Idarraga J., ${ }^{62}$, Iengo P. ${ }^{68,38}$, Igonkina O., ${ }^{69}$, Ikeno M., ${ }^{46}$, Ishikawa A., ${ }^{47}$, Ishino M., ${ }^{89}$, Iwasaki H., ${ }^{46}$, Izzo V., ${ }^{68}$, Jain V., ${ }^{43}$, Jez P., ${ }^{64}$, Jimenez Otero S., ${ }^{14}$, Johansen M., ${ }^{86}$, Johns K., ${ }^{3}$, Jones G., ${ }^{58}$, Joos M., ${ }^{14}$, Kadosaka T., ${ }^{47}$, Kajomovitz E.,${ }^{87}$, Kalinowski A., ${ }^{78}$, Kama S., ${ }^{21}$, Kanaya N.., ${ }^{89}$, Kanega F., ${ }^{89}$, Kaushik V., ${ }^{3}$, Kawagoe K., ${ }^{47}$, Kawamoto T. ${ }^{89}$, Kazarov A., ${ }^{73}$, Kehoe R., ${ }^{83}$, Kessoku K., ${ }^{89}$, Khoriauli G., ${ }^{78}$, Kieft G., ${ }^{6}$, Kirk J., ${ }^{77}$, Kiyamura H., ${ }^{47}$, Klofver P., ${ }^{14}$, Klous S., ${ }^{69}$, Kluge E.-E., ${ }^{31}$, Kobayashi T., ${ }^{89}$, Koeneke K., ${ }^{21}$, Kolos S., ${ }^{91,73}$, Kono T., ${ }^{14}$, Konoplich R., ${ }^{65}$, Konstantinidis N., ${ }^{92}$, Korcyl K., ${ }^{19}$, Ko- 
rdas K., ${ }^{7}$, Kotov V., ${ }^{24}$, Kowalewski R.V., ${ }^{98}$, Krasznahorkay A., ${ }^{14,22}$, Kreisel A., ${ }^{88}$, Kubota T., ${ }^{89}$, Kugel A., ${ }^{32}$, Kurashige H., ${ }^{47}$, Kwee R., ${ }^{35}$, Landon M., ${ }^{76}$, Lane J., ${ }^{58}$, Lankford A.J., ${ }^{91}$, Leahu L., ${ }^{12,14}$, Leahu M., ${ }^{12}$, LeCompte T., ${ }^{1}$, Ledroit F., ${ }^{29}$, Lehmann Miotto G.. ${ }^{14}$, Lei X., ${ }^{3}$, Lellouch D.,${ }^{99}$, Lendermann V., ${ }^{31}$, Levinson L., ${ }^{9}$, Li S., ${ }^{21}$, Liberti B., ${ }^{81,42}$, Lilley J.N., ${ }^{8}$, Lim H., ${ }^{1}$, Linnemann J.T. ${ }^{56}$, Lohse T., ${ }^{35}$, Losada M., ${ }^{9}$, Lowe A., ${ }^{43}$, Luci C. ${ }^{80,41}$, Luminari L.,${ }^{41}$, Lundberg J., ${ }^{14}$, Lupu N., ${ }^{87}$, Mackeprang R., ${ }^{14}$, Maettig S., ${ }^{30,21}$, Mahboubi K., ${ }^{31}$, Mahout G., ${ }^{8}$, Maltrana D.,${ }^{84}$, Mamuzic J., ${ }^{21}$, Männer R., ${ }^{32}$, Mapelli L., ${ }^{14}$, Marchese F., ${ }^{81,41}$, Marino C., ${ }^{43}$, Martin B., ${ }^{14}$, Martin B.T., ${ }^{56}$, Martyniuk A. ${ }^{58}$, Marzano F., ${ }^{41}$, Masik J. ${ }^{58}$, Matsushita T. ${ }^{47}$, McMahon T., ${ }^{34}$, Mcpherson R.,${ }^{98}$, Medinnis M. ${ }^{21}$, Meessen C., ${ }^{59}$, Meier K., ${ }^{31}$, Meirosu C., ${ }^{12}$, Merola L. ${ }^{68,38}$, Messina A., ${ }^{14}$, Middleton R.P., ${ }^{77}$, Mikenberg G., ${ }^{99}$, Mincer A., ${ }^{65}$, Mineev M., ${ }^{24}$, Misiejuk A.,${ }^{34}$, Moa T., ${ }^{86}$, Moenig K., ${ }^{21}$, Monticelli F., ${ }^{50}$, Mora Herrera C.,${ }^{26}$, Moreno D.,${ }^{9}$, Morettini P., ${ }^{27}$, Morris J.D.,${ }^{76}$, Müller F., ${ }^{31}$, Murillo Garcia R., ${ }^{91}$, Nagano K. ${ }^{46}$, Nagasaka Y., ${ }^{33}$, Nakatsuka H., ${ }^{47}$, Navarro G.A., ${ }^{9}$, Negri A., ${ }^{74,39}$, Nemethy P., ${ }^{65}$, Neusiedl A. ${ }^{57}$, Nisati A., ${ }^{41}$, Niwa T., ${ }^{47}$, Nomoto H., ${ }^{89}$, Nozaki M., ${ }^{46}$, Nozicka M., ${ }^{21}$, Nurse E., ${ }^{92}$, Ochi A., ${ }^{47}$, Oda S., ${ }^{89}$, Ohm C., ${ }^{86}$, Okumura Y., ${ }^{67}$, Oltmann B., ${ }^{57}$, Olvito D., ${ }^{75}$, Omachi C. ${ }^{47}$, Osculati B., ${ }^{27}$, Oshita H., ${ }^{85}$, Osuna C., ${ }^{5}$, Owen M.A., ${ }^{58}$, Özcan E., ${ }^{92}$, Ozone K., ${ }^{46}$, Padilla C., 5 , Panes B., ${ }^{84}$, Panikashvili N., ${ }^{61}$, Parodi F. ${ }^{27}$, Pasqualucci E., ${ }^{41}$, Pastore F., ${ }^{14}$, Patricelli S. ${ }^{68,38}$, Pauly T., ${ }^{14}$, Perantoni M., ${ }^{79}$, Perera V.J.O., ${ }^{77}$, Perez E., ${ }^{5}$, Perez Reale V., ${ }^{18}$, Perrino R., ${ }^{52,37}$, Pessoa Lima Junior H., ${ }^{79}$, Petcu M., ${ }^{11}$, Petersen B., ${ }^{14}$, Petersen J., ${ }^{14}$, Petrolo E., ${ }^{80,41}$, Piegaia R., ${ }^{13}$, Pilcher J., ${ }^{16}$, Pinzon G.. ${ }^{9}$, Policicchio A., ${ }^{15}$, Polini A., ${ }^{10,36}$, Pope B.G., ${ }^{56}$, Potter C., ${ }^{60}$, Prieur D.P.F., ${ }^{77}$, Primavera M., ${ }^{37}$, Prokoshin F., ${ }^{97}$, Qian W., ${ }^{77}$, Quinonez F., ${ }^{84}$, Rajagopalan S., ${ }^{4}$, Ramos Dos Santos Neves R., ${ }^{49}$, Reinherz E., ${ }^{88}$, Reinsch A., ${ }^{70}$, Renkel P., ${ }^{83}$, Rescigno M., ${ }^{41}$, Rieke S., ${ }^{57}$, Risler C., ${ }^{35}$, Riu I., ${ }^{5}$, Robertson S., ${ }^{60}$, Roda C., ${ }^{40}$, Rodriguez D., ${ }^{9}$, Rogriquez Y., ${ }^{9}$, Roich A., ${ }^{99}$, Romeo G., ${ }^{13}$, Rosati S., ${ }^{80,41}$, Rühr F., ${ }^{31}$, Ruiz-Martinez A., ${ }^{96}$, Ryabov Y., ${ }^{73}$, Ryan P., ${ }^{56}$, Saavedra A. ${ }^{93}$, Sakamoto H., ${ }^{89}$, Salamon A., ${ }^{81,42}$, Salnikov A., ${ }^{82}$, Salvatore D., ${ }^{15}$, Salvatore F., ${ }^{34}$, Sankey D.P.C., ${ }^{77}$, Santamarina C., ${ }^{60}$, Santonico R. ${ }^{81,42}$, Sarkisyan-Grinbaum E.,${ }^{94}$, Sasaki O., ${ }^{46}$, Savu D., ${ }^{14}$, Scannicchio D., ${ }^{14}$, Schäfer U., ${ }^{57}$, Schiavi C., ${ }^{27}$, Schlereth J., ${ }^{1}$, Schmitt K., ${ }^{31}$, Schmitt S., ${ }^{21}$, Scholtes I., ${ }^{14}$, Schooltz D. ${ }^{56}$, Schroer N., ${ }^{32}$, Schultz-Coulon H.-C., ${ }^{31}$, Schwienhorst R., ${ }^{56}$, Scott W., ${ }^{77}$, Segura E., ${ }^{5}$, Sekhniaidze G., ${ }^{38}$, Sherman D., ${ }^{14}$, Shimojima M., ${ }^{66}$, Sidoti A., ${ }^{35}$, Silverstein D., ${ }^{82}$, Silverstein S., ${ }^{86}$, Sinev N., ${ }^{70}$, Siragusa G., ${ }^{57}$, Sivoklokov S., ${ }^{63}$, Sjoen R., ${ }^{6}$, Sjölin J., ${ }^{86}$, Sloper J.E., ${ }^{14}$, Smizanska M., ${ }^{51}$, Soffer A., ${ }^{88}$, Soloviev I., ${ }^{73}$, Spagnolo S. ${ }^{52,37}$, Spiwoks R., ${ }^{14}$, Staley R.J., ${ }^{8}$, Stamen R., ${ }^{31}$, Stancu S., ${ }^{91,12}$, Steinberg P., ${ }^{4}$, Stelzer J., ${ }^{21}$, Stockton M.C., ${ }^{8}$, Stradling A., ${ }^{100}$, Straessner A., ${ }^{23}$, Strom D., ${ }^{70}$, Su D., ${ }^{82}$, Sugaya Y., ${ }^{71}$, Sugimoto T., ${ }^{67}$, Sushkov S.,, , Sutton M., ${ }^{92}$, Suzuki Y., ${ }^{46}$, Szymocha T., ${ }^{19}$, Taffard A., ${ }^{91}$, Takahashi Y., ${ }^{67}$, Takeda H., ${ }^{47}$, Takeshita T., ${ }^{85}$, Tamsett M., ${ }^{34}$, Tan C.L.A., ${ }^{8}$, Tanaka S., ${ }^{46}$, Tapprogge S., ${ }^{57}$, Tarem S., ${ }^{87}$, Tarem Z., ${ }^{87}$, Taylor C., ${ }^{92}$, Taylor R.P., ${ }^{98}$, Teixeira-Dias P., ${ }^{34}$, Thomas J.P., ${ }^{8}$, Thompson P.D., ${ }^{8}$, Tokushuku K., ${ }^{46}$, Tollefson K., ${ }^{56}$, Tomoto M., ${ }^{67}$, Topfel C., ${ }^{7}$, Torrence E. ${ }^{70}$, Touchard F., ${ }^{59}$, Tremblet L., ${ }^{14}$, Tripiana M., ${ }^{50}$, Tsuno S., ${ }^{46}$, Ünel G., ${ }^{91}$, Urquijo P., ${ }^{26}$, Urrejola P. ${ }^{84}$, Usai G.., ${ }^{16}$, Vachon B., ${ }^{60}$, Vandelli W., ${ }^{14}$, Vari R., ${ }^{80,41}$, Vaz Gil Lopes L., ${ }^{17}$, Veneziano S., ${ }^{80,41}$, Ventura A., ${ }^{52,37}$, Vercesi V., ${ }^{74}$, Vermeulen J.C., ${ }^{69,2}$, Von Der Schmitt J., ${ }^{55}$, Vorwerk V., ${ }^{5}$, Wang M., ${ }^{78}$, Watkins P.M., ${ }^{8}$, Watson A.T., ${ }^{8}$, Weber P., ${ }^{31}$, Weidberg T., ${ }^{72}$, Wengler T., ${ }^{58}$, Werner P., ${ }^{14}$, Werth M., ${ }^{91}$, Wessels M.,${ }^{31}$, Wheeler-Ellis S.. ${ }^{91}$, Whiteson D., ${ }^{91}$, Wickens F.J., ${ }^{77}$, Wiedenmann W., ${ }^{100}$, Wielers M., ${ }^{77}$, Wildt M., ${ }^{57}$, Wilkens H., ${ }^{14}$, Winklmeier F., ${ }^{14}$, Wu S.-L., ${ }^{100}$, Wu X. ${ }^{26}$, Xella S. ${ }^{64}$, Yamazaki Y.., ${ }^{47}$, Yang U., ${ }^{58}$, Yasu Y., ${ }^{46}$, Zanello L., ${ }^{80,41}$, Zema F., ${ }^{14}$, Zhang J., ${ }^{1}$, Zhao L., ${ }^{65}$, Zobernig H., ${ }^{100}$, zur Nedden M. $.{ }^{35} / /$ 
${ }^{1}$ Argonne National Laboratory, Argonne, Illinois

${ }^{2}$ University of Amsterdam, Amsterdam

${ }^{3}$ University of Arizona, Tucson, Arizona

${ }^{4}$ Brookhaven National Laboratory (BNL), Upton, New York

${ }^{5}$ Institut de Física d'Altes Energies (IFAE), Universitat Autònoma de Barcelona, Bellaterra (Barcelona)

${ }^{6}$ University of Bergen, Bergen

${ }^{7}$ Laboratory for High Energy Physics, University of Bern, Bern

${ }^{8}$ School of Physics and Astronomy, The University of Birmingham, Birmingham

${ }^{9}$ Universidad Antonio Narino, Bogotá, Colómbia

${ }^{10}$ Università di Bologna, Dipartimento di Fisica, Bologna

${ }^{11}$ National Institute of Physics and Nuclear Engineering, Bucharest

12 UPB - 'Politehnica' University of Bucharest, Bucharest

${ }^{13}$ Universidad de Buenos Aires, Buenos Aires

${ }^{14}$ European Laboratory for Particle Physics (CERN), Geneva

${ }^{15}$ Dipartimento di Fisica dell' Università della Calabria e I.N.F.N., Cosenza

${ }^{16}$ University of Chicago, Enrico Fermi Institute, Chicago, Illinois

${ }^{17}$ Laboratório de Instrumentaçao e Fisica Experimental de Particulas, Coimbra

${ }^{18}$ Nevis Laboratory, Columbia University, Irvington, New York

${ }^{19}$ Institute of Nuclear Physics, Polish Academy of Sciences, Cracow

${ }^{20}$ Faculty of Physics and Applied Computer Science, AGH University of Science and Technology,

Cracow

${ }^{21}$ Deutsches Elektronen-Synchrotron (DESY), Hamburg and Zeuthen

${ }^{22}$ University of Debrecen

${ }^{23}$ Technical University Dresden, Dresden

24 Joint Institute for Nuclear Research, Dubna

${ }^{25}$ Laboratori Nazionali di Frascati dell' I.N.F.N., Frascati

${ }^{26}$ Section de Physique, Université de Genève, Geneva

${ }^{27}$ Dipartimento di Fisica dell' Università di Genova e I.N.F.N., Genova

${ }^{28}$ Department of Physics and Astronomy, University of Glasgow, Glasgow

${ }^{29}$ Laboratoire de Physique Subatomique et de Cosmologie de Grenoble (LPSC), IN2P3-CNRSUniversité Joseph Fourier, Grenoble

${ }^{30}$ University of Hamburg, Germany

${ }^{31}$ Kirchhoff Institut für Physik, Universität Heidelberg, Heidelberg

32 ZITI Ruprecht-Karls-University Heidelberg

${ }^{33}$ Hiroshima Institute of Technology, Hiroshima

${ }^{34}$ Department of Physics, Royal Holloway, University of London, Egham

${ }^{35}$ Institut für Physik, Humboldt-Universität zu Berlin

${ }^{36}$ I.N.F.N. Bologna

${ }^{37}$ I.N.F.N. Lecce

${ }^{38}$ I.N.F.N. Napoli

${ }^{39}$ I.N.F.N. Pavia 
${ }^{40}$ Dipartimento di Fisica dell' Università di Pisa e I.N.F.N., Pisa

${ }^{41}$ I.N.F.N. Roma

${ }^{42}$ I.N.F.N. Tor Vergata

${ }^{43}$ Indiana University, Bloomington, Indiana

${ }^{44}$ nstitute for Astro- and Particle Physics, Leopold-Franzens-Universit Innsbruck

${ }^{45}$ Iowa State University, Ames, Iowa

${ }^{46}$ KEK, High Energy Accelerator Research Organisation, Tsukuba

${ }^{47}$ Kobe University, Kobe

${ }^{48}$ Laboratoire d'Annecy-le-Vieux de Physique des Particules (LAPP), IN2P3-CNRS, Annecy-leVieux

${ }^{49}$ Laboratorio de Instrumentaçao e Fisica Experimental, Lisboa

${ }^{50}$ Universidad Nacional de La Plata, La Plata

${ }^{51}$ Department of Physics, Lancaster University, Lancaster

${ }^{52}$ Università degli Studi del Salento, Dipartimento di Fisica, Lecce

${ }^{53}$ University Católica-Figueira da Foz and University Nova de Lisboa, Lisbon

${ }^{54}$ University of Liverpool, Liverpool

${ }^{55}$ Max-Planck-Institut für Physik, München

${ }^{56}$ Michigan State University, Department of Physics and Astronomy, East Lansing, Michigan

${ }^{57}$ Institut für Physik, Universität Mainz, Mainz

${ }^{58}$ School of Physics and Astronomy, University of Manchester, Manchester

${ }^{59}$ Centre de Physique des Particules de Marseille, IN2P3-CNRS, Marseille

${ }^{60}$ Department of Physics, McGill University, Montreal

${ }^{61}$ University of Michigan, Department of Physics, Ann Arbor, Michigan

${ }^{62}$ University of Montreal, Montreal

${ }^{63}$ Moscow State University, Moscow

${ }^{64}$ Niels Bohr Institute, University of Copenhagen, Copenhagen

${ }^{65}$ Department of Physics, New York University, New York

${ }^{66}$ Nagasaki Institute of Applied Science, Nagasaki

${ }^{67}$ Nagoya University, Nagoya

${ }^{68}$ Università di Napoli 'Federico II', Dipartimento di Scienze Fisiche, Napoli

${ }^{69}$ Nikhef National Institute for Subatomic Physics, Amsterdam

${ }^{70}$ University of Oregon, Eugene, Oregon

${ }^{71}$ Osaka University, Osaka

72 Department of Physics, Oxford University, Oxford

${ }^{73}$ Petersburg Nuclear Physics Institute (PNPI), St. Petersburg

${ }^{74}$ Università di Pavia, Dipartimento di Fisica Nucleare e Teorica, Pavia

75 Department of Physics, University of Pennsylvania, Philadelphia, Pennsylvania

${ }^{76}$ Physics Department, Queen Mary, University of London, London

${ }^{77}$ STFC Rutherford Appleton Laboratory, Harwell Science and Innovation Campus, Didcot, Oxon

${ }^{78}$ University of Regina, Regina

${ }^{79}$ Universidade Federal do Rio de Janeiro, COPPE/EE/IF, Rio de Janeiro

${ }^{80}$ Università di Roma I 'La Sapienza',Dipartimento di Fisica, Roma

${ }^{81}$ Dipartimento di Fisica dell' Università di Roma II 'Tor Vergata' 
${ }^{82}$ Stanford Linear Accelerator Center (SLAC), Stanford

${ }^{83}$ Department of Physics, Southern Methodist University, Dallas, Texas

${ }^{84}$ Pontificia Universidad Católica, Santiago

${ }^{85}$ Faculty of Science, Shinshu University, Matsumoto

${ }^{86}$ Fysikum, Stockholm University, Stockholm

${ }^{87}$ Department of Physics, Technion, Haifa

${ }^{88}$ School of Physics and Astronomy, Tel Aviv University, Tel Aviv

${ }^{89}$ International Center for Elementary Particle Physics, University of Tokyo, Tokyo

${ }^{90}$ Physics Department, Tokyo Metropolitan University, Tokyo

${ }^{91}$ Department of Physics and Astronomy, University of California, Irvine, California

92 Department of Physics and Astronomy, University College London, London

${ }^{93}$ University of Sydney, Sydney

${ }^{94}$ Department of Physics, The University of Texas at Arlington, Arlington, Texas

${ }^{95}$ Université de Technologie de Belfort-Montbeliard

${ }^{96}$ Instituto de Fisica Corpuscular (IFIC) Universidad de Valencia

${ }^{97}$ Universidad Técnica Federico Santa María, Valparaíso

${ }^{98}$ University of Victoria, Victoria

${ }^{99}$ Department of Particle Physics, The Weizmann Institute of Science, Rehovot

${ }^{100}$ Department of Physics, University of Wisconsin, Madison, Wisconsin

${ }^{101}$ Università di Napoli 'Parthenope' 